\title{
Short communication: Nutrient consumption patterns of Lactobacillus acidophilus KLDS 1.0738 in controlled pH batch fermentations
}

\author{
Xuepeng Lv, Gefei Liu, Xiaomei Sun, Hongyu Chen, Jiahui Sun, and Zhen Feng ${ }^{1}$ \\ Key Laboratory of Dairy Science, Ministry of Education, College of Food Science, Northeast Agricultural University, 59 Mucai Road, Harbin, \\ Heilongjiang, 150030, China
}

\begin{abstract}
This work focused on elucidating the nutrient consumption patterns of Lactobacillus acidophilus to guide the design of media for high-cell-density culture. We investigated the nutrient consumption patterns of $L$. acidophilus KLDS 1.0738 in chemically defined media in controlled $\mathrm{pH}$ batch fermentations. The most abundantly consumed amino acids, vitamins, ions, and purines and pyrimidines were Glu and Gly, pyridoxine and nicotinamide, $\mathrm{K}^{+}$and $\mathrm{PO}_{4}{ }^{3-}$, and guanine and uracil, respectively. The highest consumption rates for amino acids, vitamins, ions, and purines and pyrimidines were Asp and Arg, folic acid and pyridoxine, $\mathrm{Fe}^{2+}$ and $\mathrm{Mn}^{2+}$, and uracil and thymine, respectively. Furthermore, most of the amino acids, as well as guanine, thymine, pyridoxine, folic acid, nicotinamide, $\mathrm{Mg}^{2+}, \mathrm{PO}_{4}{ }^{3-}$, and $\mathrm{K}^{+}$had the highest bioavailability from the end of the lag growth phase to the mid-exponential growth phase. The overall consumption of glucose, adenine nucleotides, 2'-deoxyguanosine monohydrate, calcium pantothenate, $\mathrm{Fe}^{2+}$ and $\mathrm{Mn}^{2+}$ decreased with increasing average growth rate, indicating more effective use of these nutritional components at a higher average growth rate, as biomass yield based on nutritional component consumption increased. Our findings help to formulate complex media for high-cell-density cultivation and provide a theoretical basis for L. acidophilus feeding strategies.
\end{abstract}

Key words: Lactobacillus acidophilus, nutrient consumption, pattern, controlled $\mathrm{pH}$

\section{Short Communication}

Lactobacillus acidophilus is widely used in the dairy industry as a starter culture and probiotic ingredient to obtain high-quality fermented products (Batista et al.,

Received January 17, 2017.

Accepted March 29, 2017

${ }^{1}$ Corresponding author: NEAU_FengZhen@163.com
2015; Felicio et al., 2016). With rising consumption of probiotic functional foods, the high-cell-density culture of Lactobacillus is becoming increasingly important (Dong et al., 2014). For lactic acid bacteria (LAB), the ideal medium plays a major role in high-celldensity culture. Lactobacilli are fastidious organisms with complex nutrient requirements. For growth, they require carbohydrates, AA, vitamins, and nucleotides in a chemically defined medium (Hébert et al., 2004). The design of media for high-cell-density culture is founded on nutritional consumption patterns, and designing media for $L$. acidophilus requires a thorough understanding of those patterns. However, systematic research of the nutritional consumption patterns and growth of L. acidophilus has not been reported, and detailed information about its consumption of specific AA, glucose, vitamins, ions, purines, and pyrimidines is inadequate. This lack of reliable information hampers the optimization of bioprocesses and the design of cultivation media (Lahtvee et al., 2011).

The aim of the present study was to evaluate the nutrient consumption patterns of L. acidophilus KLDS 1.0738. The overall objective was to provide a theoretical foundation for designed cultivation media and feeding strategies for L. acidophilus.

We used L. acidophilus KLDS 1.0738 from a previous study (Yun et al., 2016) and subcultured it in a 250-mL Erlenmeyer flask containing $50 \mathrm{~mL}$ of de Man, Rogosa, Sharpe (MRS) broth for $16 \mathrm{~h}$ at $37^{\circ} \mathrm{C}$. Batch fermentations were conducted using a 10-L Biotech-7000 bioreactor (Shanghai Baoxing, Shanghai, China) containing 8 L of chemically defined medium. The chemically defined medium was prepared according to the method of Morishita et al. (1981), and cells were harvested by centrifugation $\left(10,000 \times g, 10 \mathrm{~min}, 4^{\circ} \mathrm{C}\right)$. The pellet was washed twice with PBS (50 mmol/L, pH 6.5), and the culture was inoculated into the chemically defined medium. Batch fermentations were carried out at a rotation speed of $200 \mathrm{rpm}, 37^{\circ} \mathrm{C}, \mathrm{pH} 5.98$. The supernatants were obtained by centrifugation $(10,000$ $\times g, 10 \mathrm{~min}, 4^{\circ} \mathrm{C}$ ) and preserved at $-80^{\circ} \mathrm{C}$ until further analysis. Batch fermentations were repeated 5 times. 
Growth rate was evaluated by spectrophotometric measurement at $650 \mathrm{~nm}$. Biomass dry weight was determined gravimetrically. Viable cell count was determined by the standard dilution method using MRS agar. Amino acids were measured using an amino acid analyzer (Acquity UPLC; Waters Corp., Milford, MA). Glucose was quantified according to the method of Asif et al. (2016). Folic acid, pyridoxine, nicotinamide, riboflavin, and calcium pantothenate were measured by HPLC (Heudi et al., 2005). Guanine, 2'-deoxyguanosine monohydrate, thymine, and uracil were measured by liquid chromatography-tandem mass spectrometry (LC-MS/MS) according to the method of Stentoft et al. (2014). Adenine nucleotides were also measured by LCMS/MS, according to the method of Lorenzetti et al. (2007). Finally, $\mathrm{Fe}^{2+}, \mathrm{K}^{+}, \mathrm{Mg}^{2+}, \mathrm{Mn}^{2+}$, and $\mathrm{PO}_{4}{ }^{3-}$ were measured using inductively coupled plasma-atomic emission spectrometry (Tu et al., 2013).

Consumption patterns of 17 AA, 5 vitamins, 5 ions, and 5 purines and pyrimidines, as well as glucose, were evaluated by culture time and biomass at various average growth rates $(\boldsymbol{\mu})$. Figure 1 shows the growth curve and $\mathrm{NaOH}$ consumption profile for Lactobacillus acidophilus KLDS 1.0738.

Figures 2a to c show changes in AA concentrations by culture time. Amino acids with the highest consumption amounts were Glu (1.62 mmol/L) and Gly $(1.53 \mathrm{mmol} / \mathrm{L})$, followed by Asp $(1.15 \mathrm{mmol} / \mathrm{L})$ and Ala $(0.85 \mathrm{mmol} / \mathrm{L})$. The AA with the lowest consumption amounts were Met $(0.02 \mathrm{mmol} / \mathrm{L})$ and Cys $(0.05$ $\mathrm{mmol} / \mathrm{L}$ ), and consumption amounts for the rest ranged from 0.39 to $0.19 \mathrm{mmol} / \mathrm{L}$. For consumption rate, the most abundantly consumed amino acids were Arg (55.55\%), Tyr (54.39\%), and Asp (52.23\%), followed by Lys $(50.57 \%)$ and Leu (49.59\%). Lowest consumption rates were for Met (12.74\%) and Cys (8.4\%), and rates for the rest ranged from 32 to $46 \%$. Figures $2 \mathrm{~d}$ and e show consumption of AA with biomass at various growth rates. The consumption patterns for Asp, Thr, Ser, Ala, Met, Leu, Tyr, Phe, His, and Arg were similar, decreasing from $\mu_{1}$ to $\mu_{2}$. The efficient use of these AA increased with increasing growth rate, as biomass yield based on consumption also increased. Consumption of the same AA increased from $\mu_{2}$ to $\mu_{3}$, showing that the efficient use of these AA decreased with increasing growth rate, as biomass yield based on consumption also increased. Consumption patterns for Ile, Pro, and Gly were similar at this growth stage. Consumption of Val and Glu increased with increasing biomass from $\mu_{1}$ to $\mu_{3}$. The efficient use of these $2 \mathrm{AA}$ decreased with increasing biomass.

Figures $2 \mathrm{f}$ and $\mathrm{g}$ show changes in vitamin concentrations with culture time. Pyridoxine $(1.81 \mu \mathrm{mol} / \mathrm{L})$ and

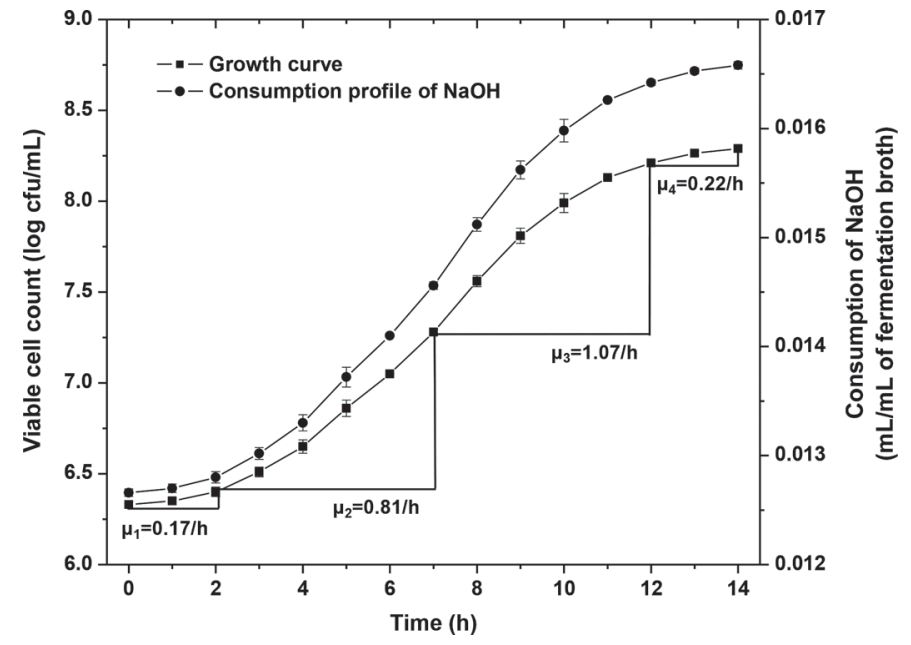

Figure 1. Growth curve and consumption profile of $\mathrm{NaOH}$ by Lactobacillus acidophilus KLDS 1.0738. $\mu$ = average growth rate. Error bars represent SE.

folic acid $(0.21 \mu \mathrm{mol} / \mathrm{L})$ had the highest and lowest consumption amounts, but the highest consumption rate was for folic acid (84.29\%). Consumption amounts for calcium pantothenate and nicotinamide were 0.42 and $1.4 \mu \mathrm{mol} / \mathrm{L}$, and rates were 20.89 and $17.05 \%$, respectively. Conversely, concentrations of riboflavin increased throughout the growth of L. acidophilus KLDS 1.0738. Figure $2 \mathrm{~h}$ shows the consumption patterns for vitamins with biomass at various growth rates. The consumption of calcium pantothenate decreased with increasing growth rates, indicating more effective use of calcium pantothenate at higher growth rates, as biomass yield based on consumption increased. Compared with other vitamins, calcium pantothenate showed the most efficient use of biomass. Consumption of pyridoxine decreased with increasing biomass from $\mu_{1}$ to $\mu_{2}$. Efficient use increased with increasing growth rates, as biomass yield based on consumption also increased. Consumption of pyridoxine increased with increasing biomass from $\mu_{2}$ to $\mu_{3}$. Efficient use of pyridoxine decreased with increasing biomass; it was highest at $\mu_{2}$ and lowest at $\mu_{1}$. Consumption patterns of folic acid and pyridoxine were similar at $\mu_{1}, \mu_{2}$, and $\mu_{3}$. Consumption patterns of niacinamide and pyridoxine were similar.

Glucose concentrations decreased sharply during the exponential growth phase (Figure 3a). Consumption amount and rate of consumption were $36.33 \mathrm{mmol} / \mathrm{L}$ and $43.68 \%$, respectively. The overall consumption of glucose decreased from 219.14 to $66.78 \mathrm{mmol} / \mathrm{g}$ of dry weight with increasing growth rates, indicating more effective use of glucose at higher growth rate values, as biomass yield based on consumption increased (Figure $3 b)$. 

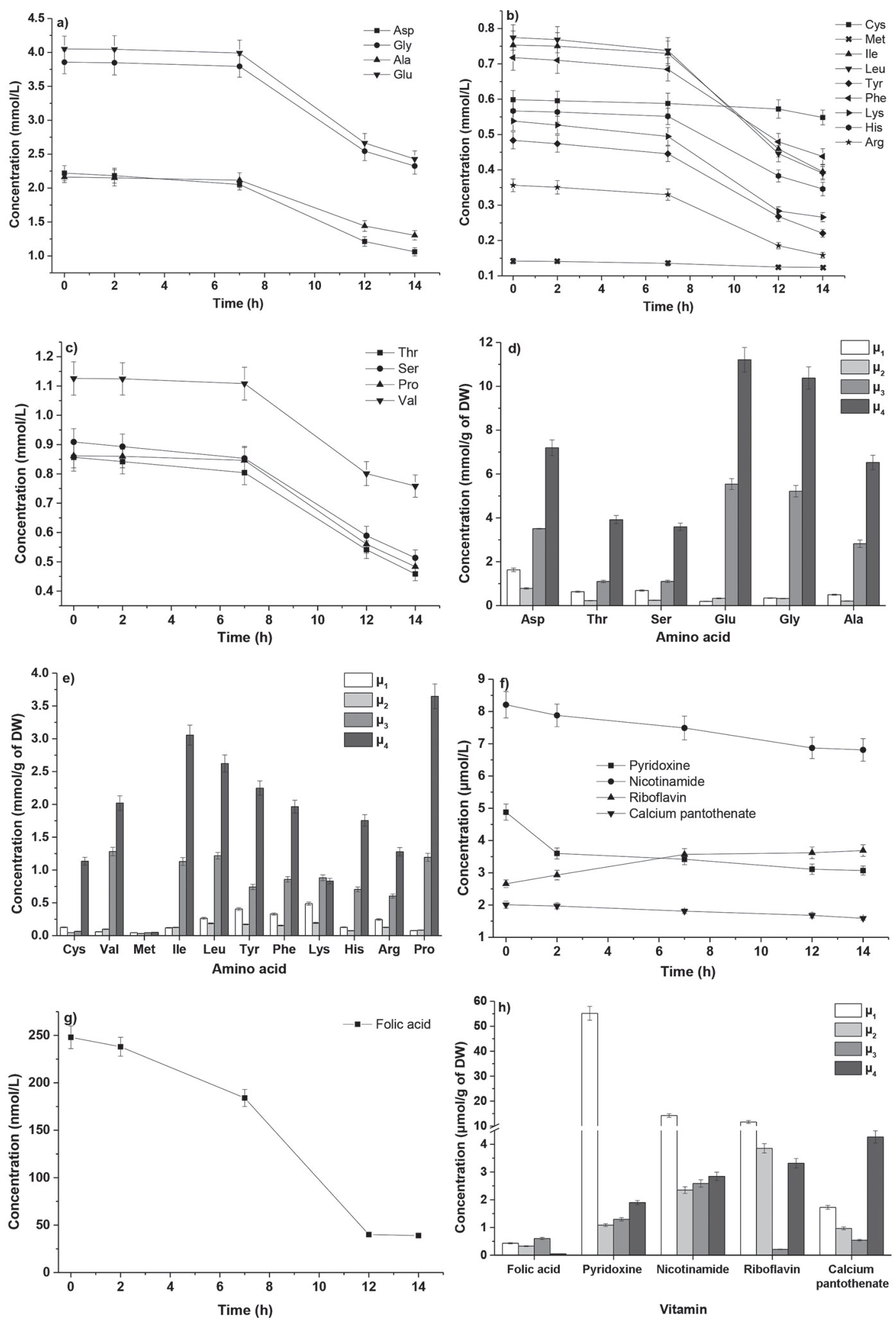

Figure 2. Residual concentrations of ( $a, b, c)$ AA and (f, g) vitamins by culture time, and consumption patterns of (d, e) AA and (h) vitamins at various average growth rates $(\mu)$. Columns represent consumption concentrations $[\mathrm{mmol} / \mathrm{g}$ of dry weight (DW)] at various average growth rates. Error bars represent SE. 

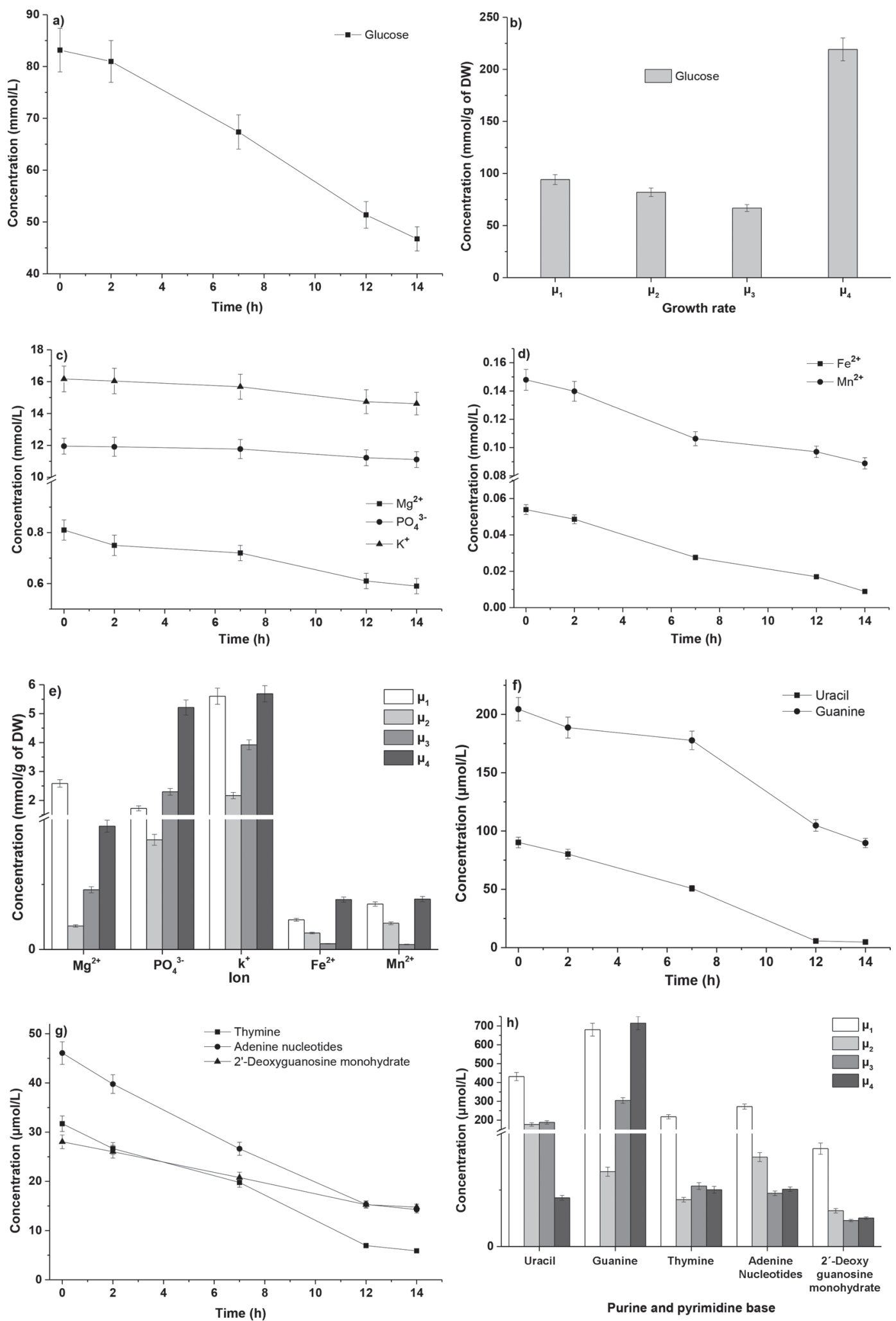

Purine and pyrimidine base

Figure 3. Residual concentrations of (a) glucose, (c, d) ions, and (f, g) purines and pyrimidines by culture time, and consumption patterns of (b) glucose, (e) ions, and (h) purines and pyrimidines at various average growth rates $(\mu)$. Columns represent consumption concentrations $[\mathrm{mmol} / \mathrm{g}$ of dry weight $(\mathrm{DW})]$ at various average growth rates. Error bars represent SE. 
Figures $3 \mathrm{c}$ and $\mathrm{d}$ show changes in ion concentrations with culture time. The ions most abundantly consumed were $\mathrm{K}^{+}(1.55 \mathrm{mmol} / \mathrm{L})$ and $\mathrm{PO}_{4}{ }^{3-}(0.84 \mathrm{mmol} / \mathrm{L})$, at rates of 9.59 and $7.02 \%$, respectively. The consumption amount and rate for $\mathrm{Mg}^{2+}$ were $0.22 \mathrm{mmol} / \mathrm{L}$ and $27.16 \%$, respectively. The consumption amount for $\mathrm{Fe}^{2+}(0.045 \mathrm{mmol} / \mathrm{L})$ was the lowest, but its consumption rate $(83.49 \%)$ was the highest. The consumption amount and rate for $\mathrm{Mn}^{2+}$ were $0.059 \mathrm{mmol} / \mathrm{L}$ and $39.91 \%$, respectively. Figure 3e shows consumption patterns for ions with biomass at various growth rates; $\mathrm{Mg}^{2+}, \mathrm{PO}_{4}{ }^{3-}$, and $\mathrm{K}^{+}$showed similar patterns at $\mu_{2}$, $\mu_{3}$, and $\mu_{4}$. Efficient use of $\mathrm{Mg}^{2+}, \mathrm{K}^{+}$, and $\mathrm{PO}_{4}{ }^{3-}$ was highest at $\mu_{2}$. Consumption of $\mathrm{Fe}^{2+}$ and $\mathrm{Mn}^{2+}$ decreased with increasing growth rate, indicating more efficient use with increasing growth rates, as biomass yield based on consumption rose. Compared with other ions, $\mathrm{Fe}^{2+}$ and $\mathrm{Mn}^{2+}$ had the highest efficient use of biomass.

Figures $3 \mathrm{f}$ and $\mathrm{g}$ show changes in concentrations of purines and pyrimidines with culture time. The consumption amount was highest for guanine (114.78 $\mu \mathrm{mol} / \mathrm{L})$, at $56.13 \%$. The consumption rate was highest for uracil (94.71\%), at $85.41 \mu \mathrm{mol} / \mathrm{L}$. The consumption amount was lowest for 2'-deoxyguanosine monohydrate $(13.27 \mu \mathrm{mol} / \mathrm{L})$, at $47.33 \%$. Consumption amounts for thymine and adenine nucleotides were 25.84 and $31.82 \mu \mathrm{mol} / \mathrm{L}$, and rates were 81.46 and $69.05 \%$, respectively. Figure 3 h shows consumption patterns for purines and pyrimidines. Uracil, guanine, and thymine showed similar consumption patterns at $\mu_{1}, \mu_{2}$, and $\mu_{3}$. The efficient use of uracil, guanine, and thymine was highest at $\mu_{2}$. The efficient use of adenine nucleotides and 2'-deoxyguanosine monohydrate was highest at $\mu_{3}$. Compared with other purines and pyrimidines, adenine nucleotides and 2'-deoxyguanosine monohydrate had the most efficient use of biomass.

Distinct ratios of nutritional components were crucial to high-cell-density cultivation of LAB. A shortage of essential nutritional components limits the growth of LAB (Sanders et al., 1999). We observed high consumption rates of folic acid, uracil, and $\mathrm{Fe}^{2+}$ during the growth of $L$. acidophilus KLDS 1.0738, and the residual concentrations of these nutritional components were very low. Their concentrations may be unfit for the growth of L. acidophilus KLDS 1.0738. Therefore, nutritional components with high consumption rates should be increased in designed cultivation media. Conversely, the concentration of riboflavin increased; it should be reduced or removed from designed cultivation media. Consumption of all $\mathrm{AA}$, as well as $\mathrm{Mg}^{2+}$, $\mathrm{K}^{+}$, and $\mathrm{PO}_{4}{ }^{3-}$, was highest at $\mu_{1}$ and $\mu_{4}$. The efficient use of these nutritional components was also lowest, because increasing biomass was not remarkable at the 2 growth stages. These findings were in agreement with Lahtvee et al. (2011).

According to Lahtvee et al. (2011), consumption of nutritional components should decrease with increasing growth rate, as biomass yield based on consumption rises. Considering consumption patterns and efficient use of glucose, $\mathrm{Fe}^{2+}, \mathrm{Mn}^{2+}$, calcium pantothenate, and 2'-deoxyguanosine monohydrate, we obtained similar results. As well, efficient use of some nutritional components should have been highest at $\mu_{3}$, except for glucose, $\mathrm{Fe}^{2+}, \mathrm{Mn}^{2+}$, calcium pantothenate, and 2'-deoxyguanosine monohydrate. Excessive consumption of these nutritional components may be used to counteract stress; accordingly, a portion of the nutritional components consumed may not have been involved in the synthesis of biomass (Huang et al., 2016). Lactic acid bacteria encounter 3 stress factors simultaneously: high concentration of lactate, high osmotic pressure, and low intracellular $\mathrm{pH}$, especially during the exponential growth phase (Yáñez et al., 2008; Rault et al., 2009; Huang et al., 2016). Amino acid catabolism in LAB plays a major role in the generation of redox power or metabolic energy, intracellular $\mathrm{pH}$ control, and resistance to stresses (Fernández and Zúñiga, 2006). Research suggests that Ala, Asp, Arg, and Glu are involved in protecting LAB against the damage of a low pH environment (SenouciRezkallah et al., 2011; Guan et al., 2013). In the present study, AA of high consumption were Glu, Gly, Ala, Asp, and Arg; these AA may help LAB resist stress. Furthermore, phosphate and exogenous purine enhance resistance to acid stress in Lactococcus lactis (Ryssel et al., 2014). Insufficient supply of these compounds may affect the efficient growth of LAB. Therefore, nutritional components with special physiological roles should be considered in cultivation media. According to nutrient consumption patterns for L. acidophilus KLDS 1.0738, the nutritional components with large consumption amount, rate, and special physiological function should be added at appropriate concentrations. Additionally, excessively high initial concentrations of nutritional components may inhibit the growth of LAB (Huang et al., 2016). The development of an efficient feeding strategy is important for preventing such inhibitory effects during high-cell-density culture of L. acidophilus KLDS 1.0738 .

Concentrations of riboflavin increased in this study, suggesting that L. acidophilus KLDS 1.0738 could synthesize riboflavin; a similar result was obtained by Ewe et al. (2010). Although L. acidophilus NCFM was found to be capable of synthesizing pantothenate (Altermann et al., 2005), calcium pantothenate showed a very low 
consumption amount and rate in the present study. Either L. acidophilus KLDS can synthesize pantothenate or its requirement for pantothenate is very low. Bacteria require and produce $\mathrm{B}$ vitamins simultaneously, because they can use and synthesize different vitamins via 2 pathways concurrently (Azcarate-Peril et al., 2008). In the present study, the requirement amounts for B vitamins with low consumption amount and rate were low, or the B vitamins were synthesized in $L$. acidophilus KLDS. Cys may be a similar with respect to $L$. acidophilus KLDS. Many lactobacilli are auxotrophic for purines and pyrimidines, and some have an additional requirement for deoxynucleoside, because they cannot reduce ribonucleotide to the corresponding deoxyribonucleotide for DNA synthesis (Kilstrup et al., 2005). In the present study, purines and pyrimidines displayed generally high consumption amounts and rates. Furthermore, the residual concentrations of guanine and thymine were particularly low; we postulate that $L$. acidophilus KLDS is auxotrophic for both purines and pyrimidines.

For the high-cell-density cultivation of L. acidophilus, consumption patterns of nutritional components are important criteria for designing cultivation media. $\mathrm{Nu}-$ tritional components with high consumption amount and rate, low residual concentrations, and special physiological functions should be increased in the media for the high-cell-density cultivation of $L$. acidophilus; in the present study, those components were Asp, Arg, Tyr, Lys, pyridoxine, folic acid, $\mathrm{Fe}^{2+}$, guanine, uracil, and thymine. Nutritional components with low consumption amount and rate, and high residual concentrations should be decreased in the media; in the present study, those components were Cys, nicotinamide, $\mathrm{K}^{+}$and $\mathrm{PO}_{4}{ }^{3-}$. Riboflavin should be reduced or removed from the media.

\section{ACKNOWLEDGMENTS}

This work was supported by grants from the Natural Science Foundation of Heilongjiang Province (C2016023), the Academic Backbone Project of Northeast Agricultural University (15XG21) and the National Natural Science Foundation of China (31571930).

\section{REFERENCES}

Altermann, E., W. M. Russell, M. A. Azcarate-Peril, R. Barrangou, B. L. Buck, O. McAuliffe, N. Souther, A. Dobson, T. Duong, M. Callanan, S. Lick, A. Hamrick, R. Cano, and T. R. Klaenhammer. 2005. Complete genome sequence of the probiotic lactic acid bacterium Lactobacillus acidophilus NCFM. Proc. Natl. Acad. Sci. USA 102:3906-3912.
Asif, A., Z. Hussain, M. Balal Arain, N. Shah, K. Mohammed Khan, H. Gulab, and A. Zada. 2016. Development of microwave assisted spectrophotometric method for the determination of glucose. Spectrochimica Acta. A Mol. Biol. 153:374-378.

Azcarate-Peril, M. A., E. Altermann, Y. J. Goh, R. Tallon, R. B. Sanozky-Dawes, E. A. Pfeiler, S. O'Flaherty, B. L. Buck, A. Dobson, T. Duong, M. J. Miller, R. Barrangou, and T. R. Klaenhammer. 2008. Analysis of the genome sequence of Lactobacillus gasseri ATCC 33323 reveals the molecular basis of an autochthonous intestinal organism. Appl. Environ. Microbiol. 74:4610-4625.

Batista, A. L. D., R. Silva, L. P. Cappato, C. N. Almada, R. K. A. Garcia, M. C. Silva, R. S. L. Raices, D. B. Arellano, A. S. Sant'Ana, C. A. Conte Junior, M. Q. Freitas, and A. G. Cruz. 2015. Quality parameters of probiotic yogurt added to glucose oxidase compared to commercial products through microbiological, physical-chemical and metabolic activity analyses. Food Res. Int. 77:627-635.

Stentoft, C., M. Vestergaard, P. Løvendahl, N. B. Kristensen, J. M. Moorby, and S. K. Jensen. 2014. Simultaneous quantification of purine and pyrimidine bases, nucleosides and their degradation products in bovine blood plasma by high performance liquid chromatography tandem mass spectrometry. J. Chromatogr. A 1356:197-210.

Felicio, T. L., E. A. Esmerino, V. A. S. Vidal, L. P. Cappato, R. K. A. Garcia, R. N. Cavalcanti, M. Q. Freitas, C. A. Conte Junior, M. C. Padilha, M. C. Silva, R. S. L. Raices, D. B. Arellano, H. M. A. Bollini, M. A. R. Pollonio, and A. G. Cruz. 2016. Physico-chemical changes during storage and sensory acceptance of low sodium probiotic Minas cheese added with arginine. Food Chem. 196:628-637.

Fernández, M., and M. Zúñiga. 2006. Amino acid catabolic pathways of lactic acid bacteria. Crit. Rev. Microbiol. 32:155-183.

Hébert, E. M., R. R. Raya, and G. S. de Giori. 2004. Nutritional requirements of Lactobacillus delbrueckii ssp. lactis in a chemically defined medium. Curr. Microbiol. 49:341-345.

Heudi, O., T. Kilinc, and P. Fontannaz. 2005. Separation of watersoluble vitamins by reversed-phase high performance liquid chromatography with ultra-violet detection: application to polyvitaminated premixes. J. Chromatogr. A 1070:49-56.

Sanders, J. W., G. Venema, and J. Kok. 1999. Environmental stress responses in Lactococcus lactis. FEMS Microbiol. Rev. 23:483-501.

Ewe, J. A., W. N. Wan-Abdullah, and M. T. Liong. 2010. Viability and growth characteristics of Lactobacillus in soymilk supplemented with B-vitamins. Int. J. Food Sci. Nutr. 61:87-107.

Lahtvee, P. J., K. Adamberg, L. Arike, R. Nahku, K. Aller, and R. Vilu. 2011. Multi-omics approach to study the growth efficiency and amino acid metabolism in Lactococcus lactis at various specific growth rates. Microb. Cell Fact. 10:12.

Kilstrup, M., K. Hammer, P. Ruhdal Jensen, and J. Martinussen. 2005. Nucleotide metabolism and its control in lactic acid bacteria. FEMS Microbiol. Rev. 29:555-590.

Guan, N., L. Liu, H. D. Shin, R. R. Chen, J. Zhang, J. Li, G. Du, Z. Shi, and J. Chen. 2013. Systems-level understanding of how Propionibacterium acidipropionici respond to propionic acid stress at the microenvironment levels: Mechanism and application. J. Biotechnol. 167:56-63.

Lorenzetti, R., S. Lilla, J. L. Donato, and G. de Nucci. 2007. Simultaneous quantification of GMP, AMP, cyclic GMP and cyclic AMP by liquid chromatography coupled to tandem mass spectrometry. J. Chromatogr. B Analyt. Technol. Biomed. Life Sci. 859:37-41.

Rault, A., M. Bouix, and C. Béal. 2009. Fermentation pH influences the physiological-state dynamics of Lactobacillus bulgaricus CFL1 during pH-controlled culture. Appl. Environ. Microbiol. 75:43744381.

Ryssel, M., A. M. Hviid, M. S. Dawish, J. Haaber, K. Haamer, J. Martinussen, and M. Kilstrup. 2014. Multi-stress resistance in Lactococcus lactis is actually escape from purine-induced stress sensitivity. Microbiology 160:2551-2559. 
Senouci-Rezkallah, K., P. Schmitt, and M. P. Jobin. 2011. Amino acids improve acid tolerance and internal $\mathrm{pH}$ maintenance in Bacillus cereus ATCC14579 strain. Food Microbiol. 28:364-372.

Huang, S., Z. W. Ai, X. M. Sun, G. F. Liu, S. Zhai, M. Zhang, H Chen, and Z. Feng. 2016. Influence of arginine on the growth, arginine metabolism and amino acid consumption profiles of Streptococcus thermophilus $\mathrm{T} 1 \mathrm{C} 2$ in controlled $\mathrm{pH}$ batch fermentations. J. Appl. Microbiol. 121:746-756.

Morishita, T., Y. Deguchi, M. Yajima, T. Sakurai, and T. Yura. 1981 Multiple nutritional requirements of lactobacilli: Genetic lesions affecting amino acid biosynthetic pathways. J. Bacteriol. 148:64-71.

Tu, Y., Y. Zhao, M. Xu, X. Li, and H. Du. 2013. Simultaneous determination of 20 inorganic elements in preserved egg prepared with different metal ions by ICP-AES. Food Anal. Methods 6:667-676.
Yáñez, R., S. Marques, F. M. Gírio, and J. C. Roseiro. 2008. The effect of acid stress on lactate production and growth kinetics in Lactobacillus rhamnosus cultures. Process Biochem. 43:356-361.

Yun, Z., A. L. Li, Y. Q. Sun, P. Du, L. B. Liu, S. Li, and C. Zhang. 2016. Lactobacillus acidophilus regulates STAT3 and STAT5 signaling in bovine $\beta$-lg-sensitized mice model. Dairy Sci. Technol. 96:501-512

Dong, Z., L. Gu, J. Zhang, M. Wang, G. Du, J. Chen, and H. Li. 2014 Optimisation for high cell density cultivation of Lactobacillus salivarius BBE 09-18 with response surface methodology. Int. Dairy J. 34:230-236. 